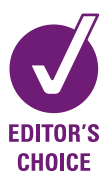

CHOICE
${ }^{1}$ New England Center for Stroke Research, Department of Radiology, University of Massachusetts Medical School, Worcester, Massachusetts, USA

${ }^{2}$ Department of Neurosurgery, Northwestern University Feinberg School of Medicine, Chicago, Illinois, USA

${ }^{3}$ Division of Neuroradiology, Department of Radiology, Northwestern University Feinberg School of Medicine, Chicago, Illinois, USA ${ }^{4}$ Research and Development, Micrus Endovascular Inc, San Jose, California, USA ${ }^{5}$ Department of Neurosurgery, School of Medicine and Biomedical Sciences, University at Buffalo, State University of New York, Buffalo, New York, USA

\section{Correspondence to} Dr M J Gounis, University of Massachusetts, 55 Lake Avenue N, Sa-107R, Worcester, MA 01655, USA

matt.gounis@umassmed.edu

Received 19 November 2010 Accepted 7 December 2010

Published Online First

24 January 2011

\title{
The impact of coil shape design on angiographic occlusion, packing density and coil mass uniformity in aneurysm embolization: an in vitro study
}

\author{
Manik Mehra, ${ }^{1}$ Michael C Hurley, ${ }^{2,3}$ Matthew J Gounis, ${ }^{1}$ Robert M King, ${ }^{1}$ \\ Ali Shaibani, ${ }^{2,3}$ Guilherme Dabus, ${ }^{2,3}$ Fatimaezzahra E Labdag, ${ }^{4}$ Elad I Levy, \\ Bernard R Bendok ${ }^{2,3}$
}

\section{ABSTRACT}

Objective To investigate the impact of coil design on the distribution of the coil mass in a controlled in vitro experiment. A secondary objective was to study the relationship between angiographic occlusion, packing density and coil mass uniformity.

Methods Seven silicon side wall aneurysm models were embolized in each arm with a different coil design under fluoroscopic guidance. Packing density calculations and scoring of the angiographic occlusion were made. The models were embedded in epoxy and sectioned through the aneurysm neck and the dome. The sections were imaged and processed to derive the fractional surface area of coil material over the coil free area, a quantitative endpoint representing the percentage of the given region of interest consumed by coils. The SD of the surface area fractions is inversely proportional to the uniformity of coil distribution.

Results The novel triangular primary wind design of the Deltapaq achieved a more homogenous distribution of coils within the aneurysm dome compared with the helical and complex microcoil systems $(p=0.018)$. The packing density achieved by the Deltapaq $(39.1 \pm 1.6 \%)$ was significantly higher than the complex $(35.2 \pm 2.8 \%)$ and the helical (32.2 $\pm 3.3 \%)$ coils. Angiographically, aneurysms coiled by the Deltapaq were more likely to obtain a Raymond score of class I.

Conclusion Evaluation of emerging coil technologies with respect to treatment durability may be well served by an assessment of their uniformity of distribution within an aneurysm in addition of the traditional packing density and angiographic occlusion scoring methods.

\section{INTRODUCTION}

Endovascular approaches have increased in importance in the management of intracranial aneurysms over the past 2 decades. ${ }^{1}{ }^{2}$ However, concerns remain regarding aneurysm recanalization after coiling with the potential for subarachnoid hemorrhage or mass effect. ${ }^{3} 4$

Aneurysm recanalization can be attributed to two factors: coil compaction and aneurysm regrowth. ${ }^{5}$ Significant factors determining the likelihood of aneurysm recanalization/coil compaction are aneurysm size, initial post-embolization angiographic outcome and packing density. Smaller aneurysms have a recanalization rate of $5-20 \%$ versus large and giant aneurysms having a 35\% and 50\% recanalization rate, respectively. ${ }^{6}$ Achieving high packing densities remain a challenge in these large volume aneurysms, and the rate of compaction observed in them is also higher. $^{3}{ }^{7}$ Incomplete initial postembolization angiographic occlusion has a very strong statistical correlation with future increases in the size of the remnant requiring retreatment. ${ }^{3} 8$ Higher packing density is inversely related to coil compaction and the rate and degree of aneurysm recurrence. $^{7}{ }^{8}$ This forms the basis of the current practice of embolizing an aneurysm as tightly as possible using more conformable and larger diameter coils to achieve a more densely packed aneurysm. ${ }^{6}$ Previous work on the modeling of the interaction between the coils within an aneurysm with hemodynamic forces suggests that lower permeability of a coil mass at a given packing density would accelerate intra-aneurysmal thrombosis due to drastic reduction in intra-aneurysmal velocities and increased residence times. ${ }^{9}$ Achieving a more homogenous coil distribution within an aneurysm even at the same packing density may enhance its performance and decrease aneurysm recanalization due to compaction. The performance of various coils vis a vis homogenous distribution of the embolic material within an aneurysm has not been well studied to date.

Previous experience in the in vitro assessment of the packing of the coiled aneurysm models, including assessment of the neck coverage and angiographic occlusion, has lead to the technique which can easily be adapted to assess microcoils under controlled conditions. ${ }^{10}$ In this study, we investigate the performance of different microcoil designs in embolizing saccular silicone aneurysm models and evaluate them with respect to angiographic occlusion, calculated packing density, macroscopic neck coverage and coil distribution/ compartmentalization.

\section{MATERIALS AND METHODS Aneurysm model, study design and coiling procedure}

Twenty-one identical berry shaped silicone aneurysm models were constructed using a technique previously described. ${ }^{11}$ The parent vessel had a diameter of $4 \mathrm{~mm}$, and the dome and neck measured $8 \mathrm{~mm}$ and $4 \mathrm{~mm}$, respectively. Each aneurysm model was connected to a pulsatile flow loop circulating with deionized water at $37^{\circ} \mathrm{C}$ The pulse pressure delivered to the circuit was set to physiological conditions at a circulation rate of $100 \mathrm{ml} / \mathrm{min}$. Seven silicone aneurysms were 
assigned to be coiled under each of the three study arms with a $0.010^{\prime \prime}$ helical (Helipaq), 0.014" complex (Cashmere) and a $0.0105^{\prime \prime}$ new triangular primary wind (Deltapaq) microcoil systems (Micrus Endovascular, San Jose, California, USA).

The coiling was performed in an angiography suite under fluoroscopic control using a $6 \mathrm{~F}$ guide catheter and identical microcatheters. The operators (BRB and $\mathrm{MCH}$ ) were blinded to the coil type being used. The aneurysms were first framed with an $8 \mathrm{~mm}$ spherical shaped coil (Micrusphere) and then embolized with the respective coil type in each arm. The size and number of coils used for packing each aneurysm were determined by the operator based on the residual space. The coiling ceased after achieving the procedural end points which were complete aneurysm obliteration, catheter dislodgement from aneurysm or herniation of coil loops into the 'parent artery'. Fluoroscopy images and videos of the coiling session were recorded. The type, size and number of coils used were documented for packing density calculations. At the end of the coiling procedure, a final control angiogram was performed for grading of any residual filling using the Raymond and Roy classification. ${ }^{12}$

To control for the potential sources of bias in the study, the aneurysms were sequentially assigned identification numbers and randomly selected for one of the three study arms for coiling. There was no statistically significant difference between the aneurysm volumes of the silicone aneurysm models used for the three study arms ( $p>0.05)$. The embolization procedure was performed by two experienced operators, each placing every second coil, using the same microcatheter type, in a randomized, blinded fashion.

\section{Data analysis}

The aneurysm volume of the silicone aneurysm model was measured by titrating deionized water into the aneurysm until the neck was reached. Five measurements were made using a $1 \mathrm{ml}$ syringe and the average volume was calculated and recorded. The volume of the coils deployed into the aneurysm was calculated by multiplying the length of the coil with the cross sectional area. ${ }^{10}$ Packing density was calculated as the ratio of the total coil volume to aneurysm volume.

Assessment of the angiographic occlusion from the digital subtracted angiogram acquired at the end of the coiling procedure using Raymond's scale, graded as class 1 (completely occluded), class 2 (persistence of any portion of the original defect of the arterial wall-residual neck) and class 3 (opacification of the aneurysmal sac-residual aneurysm) was made by three angiographic evaluators who were blinded to the coil arm. ${ }^{12}$ Each evaluator was already familiar with the Raymond scale and also provided with a graphic illustration of the grades and the typical radiographic artifacts which may be encountered, as in a true clinical case (figure 1). Evaluators were additionally instructed to allow for atypical rim-like penetration of contrast around the coil mass, a pattern not typically seen in clinical cases and likely due to the absence of clotting in the in vitro model. Luminal encroachment, defined as the percentage stenosis in the arterial model at the aneurysmal neck caused by the homogenous extension of the coil mass, disregarding the individual coil loops protruding into the lumen, was also calculated from the angiographic images.

\section{Aneurysm sectioning}

Low viscosity epoxy embedding medium Spurr's resin (SPI Supplies/Structure Probe, Inc, West Chester, Pennsylvania, USA) was prepared in the fume hood by combining its components in a beaker and stirring vigorously. The bubbles in the solution

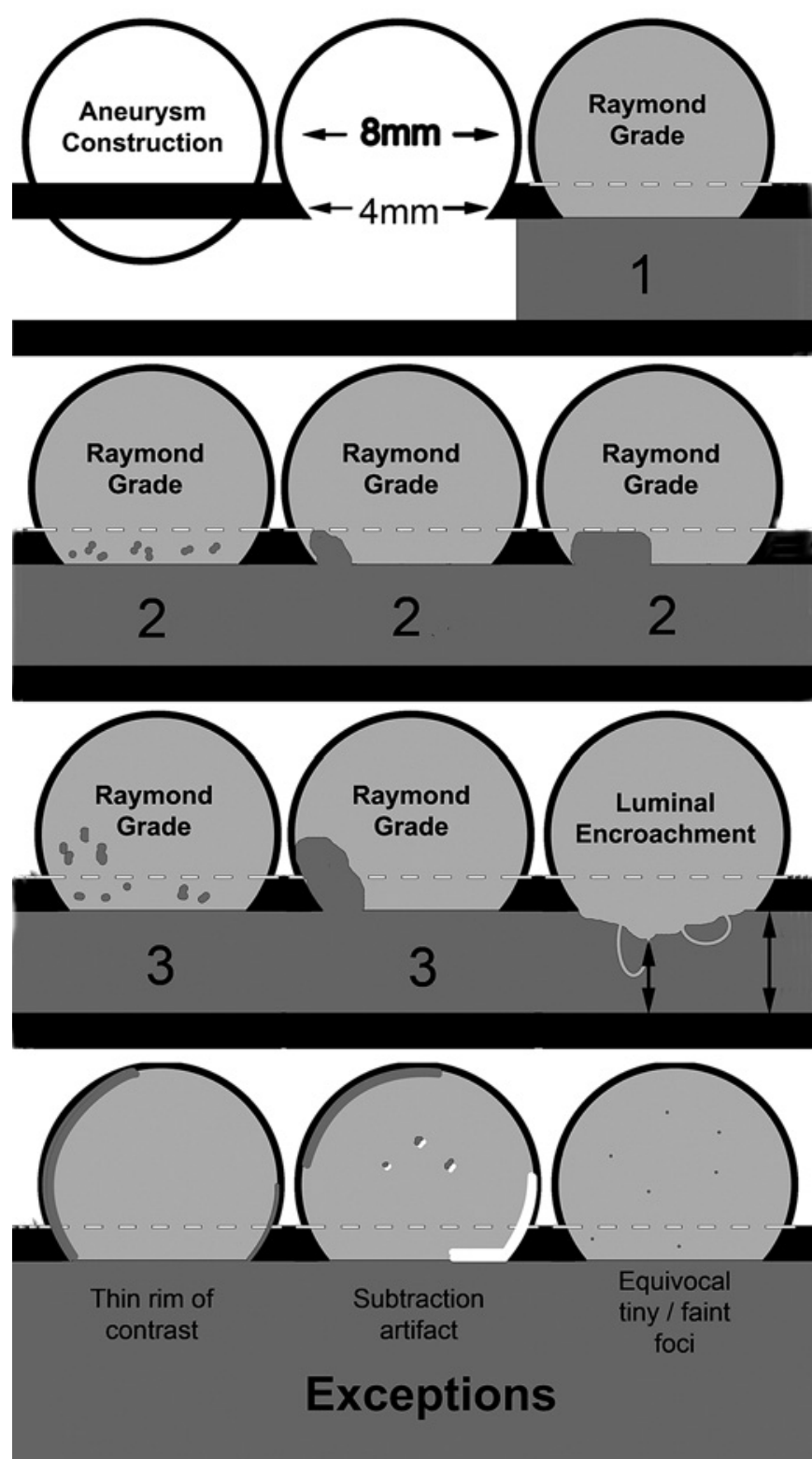

Figure 1 Illustration of the angiographic grading system. The Raymond scale grades the degree of aneurysm occlusion as: 1 -complete occlusion, 2-any filling of the neck whether interstitial, dog ear or larger, but not extending beyond the neck, and 3-filling of the aneurysm extending beyond the neck into the main sac. The neck was defined as the defect in the wall of the arterial model as this can be directly delineated on appropriately windowed fluoroscopic images. Luminal encroachment was defined as the percentage stenosis in the arterial model at the aneurysmal neck caused by protrusion of the coil mass. Individual loops were not included in the measurement but rather the homogenous extension of the coil mass. Finally, the exceptions allow the evaluator to disregard certain artifacts, including the rim-like penetration of contrast and the equivocal presence of tiny interstitial foci which are felt to be a consequence of the lack of coagulation in the in vitro model. Radiographic subtraction artifacts were also allowed for.

were extracted under vacuum. Coiled aneurysm models were infiltrated with the freshly prepared resin. The specimen immersed in the resin was cured in a vacuum chamber to improve its penetration and extract all of the residual air. The inflow and outflow zones of the specimen were marked and both ends of the vessel sealed with clips and allowed to polymerize by incubation at $60^{\circ} \mathrm{C}$ for $48 \mathrm{~h}$. A Buehler Isomet Low 
Speed Saw (Buehler, Lake Bluff, Illinois, USA) was used to section the specimen using a diamond wafering blade (Buehler Series 15HC Diamond). The first cut was made to obtain the neck section and subsequently two vertical cuts were made on either side of the medial aspect of the aneurysmal dome (figure $2 \mathrm{~A}-\mathrm{C}$ ). The sections were then mounted on the slides using cyanoacrylate super glue, ground, polished and imaged.

\section{Image analysis}

After the sections were imaged, each image was imported into Matlab (MathWorks, Natick, Massachussets, USA) for analysis. Using a graphical user interface programmed into Matlab, a mask that excludes the background was created. Next, the mask was overlaid on the image and multiplied using a binary Hadamard product; this allowed only the information within the mask to remain visible. At this point the image was viewed and a seed was planted in each coil normal to the plain of the section. The seeds were then used to grow the regions inside the coils so that they would be considered as continuous cylinders.

The resulting image was divided into radial regions of interest (ROIs). The neck sections, which were relatively small, contained eight evenly spaced ROIs constructed from lines that extended radially with $45^{\circ}$ separation. The procedure was repeated in the larger dome sections except higher precision was employed using 24 evenly distributed ROIs (figure 2D,E). Images from both the neck and dome sections were converted into an intensity image, and contrast limited adaptive histogram equalization was performed as described previously. ${ }^{10}$ Ultimately, the number of pixels from thresholding that represent the coil mass was divided by the number of pixels within the region of interest. The fractional surface area calculation provided a quantitative endpoint representing the percentage of the given ROI that was consumed by coils. For a given section, the $\mathrm{SD}$ of the surface area fractions is inversely proportional to the uniformity of coil distribution throughout the section. Therefore, the SD was subtracted from 100 for each section to provide a directly proportional uniformity index.

\section{Statistics}

For each set of data, four statistical comparisons were made using a three member analysis of variance (ANOVA) with a Student-Newman-Keuls post test. Data normality was tested using the method of Kolmogorov and Smirnov. The SDs of the populations were assumed to be equal and were tested using Bartlett's method. For categorical data, Fisher's exact test was used. An $\alpha$ value of 0.05 was chosen to be acceptable for rejection of the null hypothesis.

\section{RESULTS}

\section{Packing density}

The mean packing density calculated for the Deltapaq $(39.1 \pm 1.6 \%)$ was significantly higher than the complex $(35.2 \pm 2.8 \%)$ and the helical $(32.2 \pm 3.3 \%)$ coil systems (figure $3 \mathrm{~A})$. The greater packing density achieved by the Deltapaq was statistically different compared with the Helipaq $(p=0.001)$ and Cashmere coil systems $(p=0.012)$. The Cashmere tended to have greater packing density in comparison with the Helipaq but this result did not achieve statistical significance $(p=0.096)$.

\section{Angiographic assessment}

The angiographic assessment made independently by the three blinded evaluators was in very good agreement (intraclass correlation coefficient $=0.76$ ). The Deltapaq microcoils were more likely to provide a grade 1 occlusion score on Raymond's scale compared with the helical ( $p=0.0102$, OR 12.3, 95\% CI 1.4 to 110; Fisher's exact test) or the complex coil systems ( $p=0.0335$, OR 5.8, 95\% CI 1.1 to 32.1). In general, aneurysms appeared well packed and most remnants were of the interstitial variety. There were very few type 1 occlusions, likely due to the lack of thrombosis in the models, and those that were classified as type 1 can be inferred to have a very solid packing at the neck. The mean luminal encroachment calculated for the Deltapaq $(22.14 \pm 10.27 \%)$ was not statistically different from the helical $(20.57 \pm 9.48 \%)$ or complex $(25.85 \pm 4.45 \%)$ coil systems.
Figure 2 Illustration describing the sectioning methodology. The embedded aneurysm specimen with the inflow domain marked in red (A). The first section is taken at the neck of the aneurysm (B). The remaining dome of the aneurysm is then sectioned in the frontal plane into two equal halves. $\mathrm{A}$ section is then procured from the medial aspect of each half $(C)$. Image of a dome section (D) that is thresholded and subdivided into 24 regions of interest (E). Red surface in (D) indicates the inflow zone of the aneurysm surface.

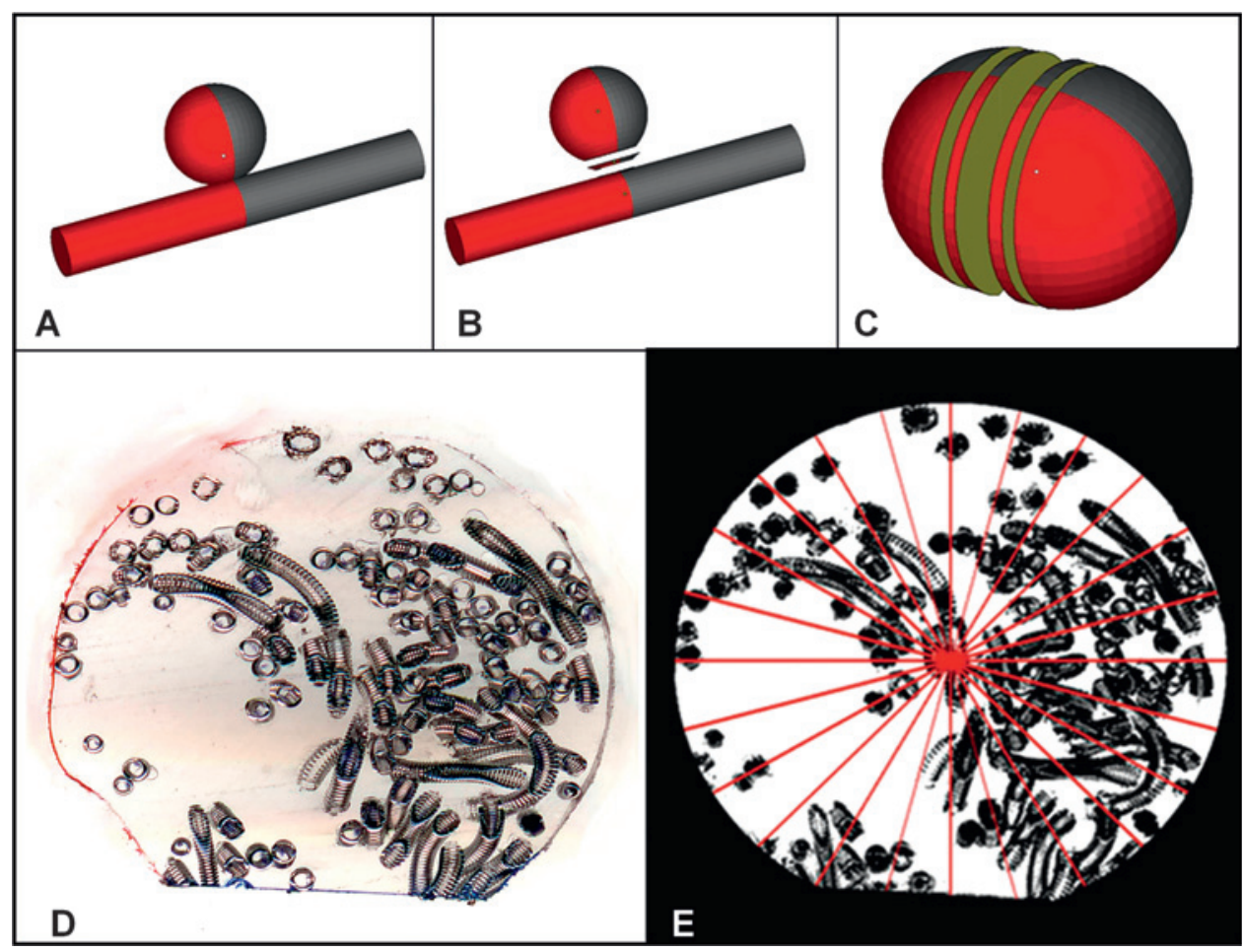


Figure 3 (A) Box and whiskers plot showing the median, maximum, minimum and upper and lower quartiles of the packing density as a function of coil type (Deltapaq (DP), Helical (HCS) and the complex coiling system (CCS)). The mean packing densities of the three arms were DP $39.1 \pm 1.6 \%$, CCS $35.2 \pm 2.8 \%$ and HCS $32.2 \pm 3.3 \%$. (B) The coil uniformity of the dome sections plotted against packing density. A proportional relationship is found $(R=0.48, p=0.029)$. The solid line is determined from a best fit linear regression analysis, and the broken lines indicate the $95 \%$ Cls.
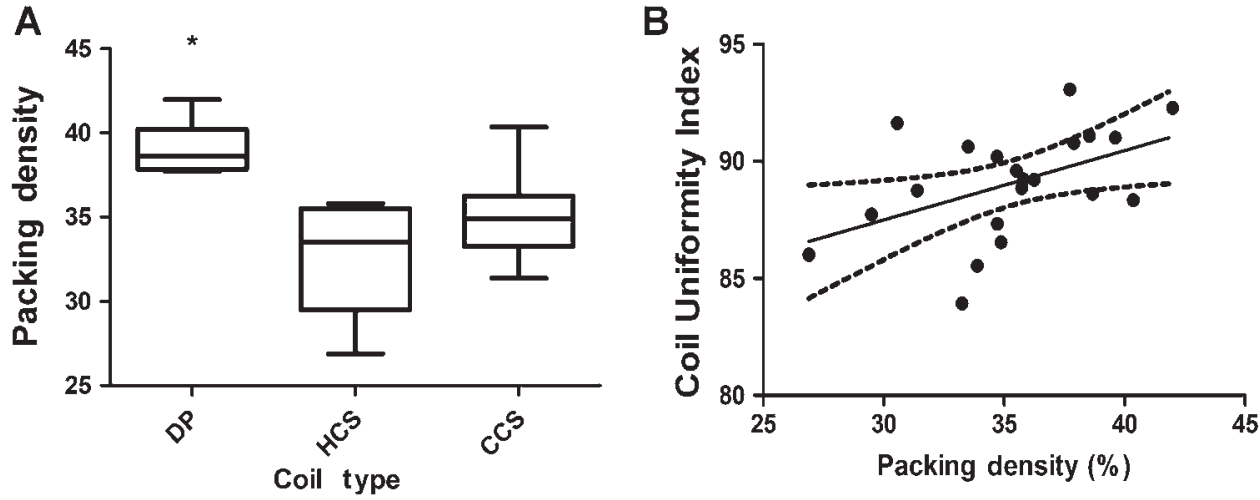

\section{Surface area fractions}

All aneurysms were successfully embedded and sectioned three times, with one neck section and two dome sections.

The surface area fractions for both the dome and neck sections were independent of coil design (figure 4A,B). On average, nearly half (range $41-49 \%$ ) of the area in these sections contained metallic coils. Furthermore, the calculated surface area fractions were not a function of packing density $(p>0.05$, the slope of the relationship was not significantly different from 0 ).

\section{Coil uniformity}

In the analysis of the dome region, it was found that the uniform distribution of coils was dependent on coil type (figure 4C). The coil system with the triangular primary wind (Deltapaq) was more uniformly distributed in the dome of the aneurysm compared with the complex and the helical coil systems ( $p=0.018$, ANOVA). Conversely, the uniformity of the neck sections was not dependent on coil type (figure 4D). The uniformity index of the dome region correlated with the packing density (figure $3 B, R=0.48, p=0.029$ ) but this relationship was not observed for the neck sections $(p>0.05)$.

\section{DISCUSSION}

The goal of endovascular embolization is to isolate and exclude the aneurysm from the cerebral circulation by decreasing the residual intra-aneurysmal blood flow, promoting thrombus organization and endothelial cell proliferation across the neck with preservation of the parent vessel. ${ }^{9} 13$ Both angiographic occlusion, a very subjective measure, and packing density, can falsely project optimal packing and overlook areas of low coil density which can provide a nidus for the coils to compact. Aneurysm recanalization and compaction can be attributed to the complex interactions between the local hemodynamics and the intra-aneurysmal flow which exert significant forces on the coil mass. Even though the overall force exerted on the coil mass is not dependent on the coil structure, the resulting intraaneurysmal flow varies with permeability which affects particle residence times and intra-aneurysmal thrombosis. ${ }^{9}$ Permeability
Figure 4 Box and whiskers plots showing the median, maximum, minimum and upper and lower quartiles of the surface area fractions and coil uniformity for the dome $(A, C)$ and neck $(B, D)$ sections, respectively, as a function of coil type (Deltapaq (DP), Helical (HCS) and the complex coiling system (CCS)). ${ }^{*} p<0.05$.

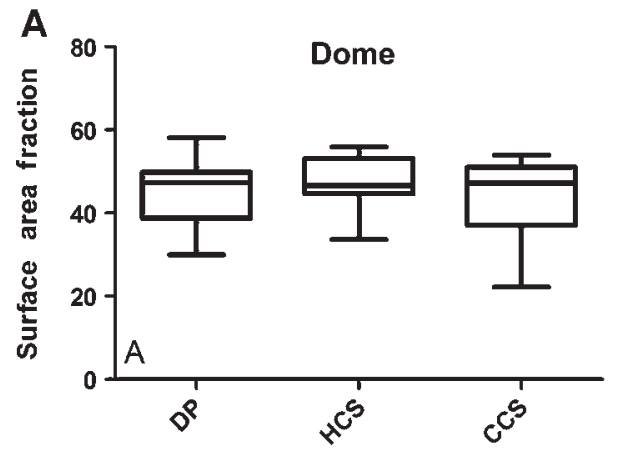

Coil type

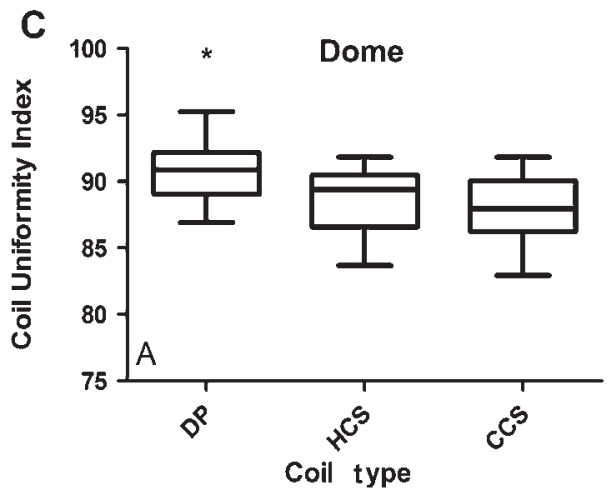

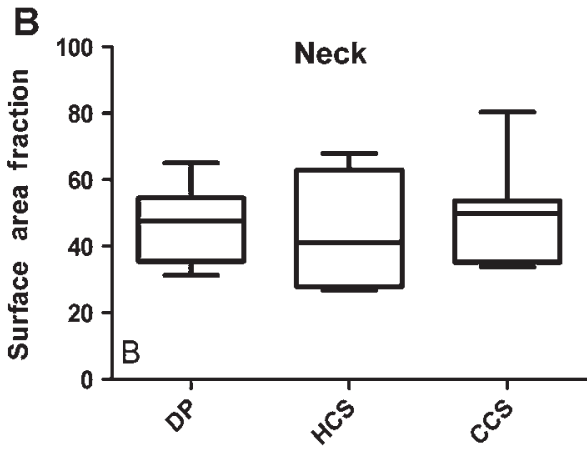

Coil type

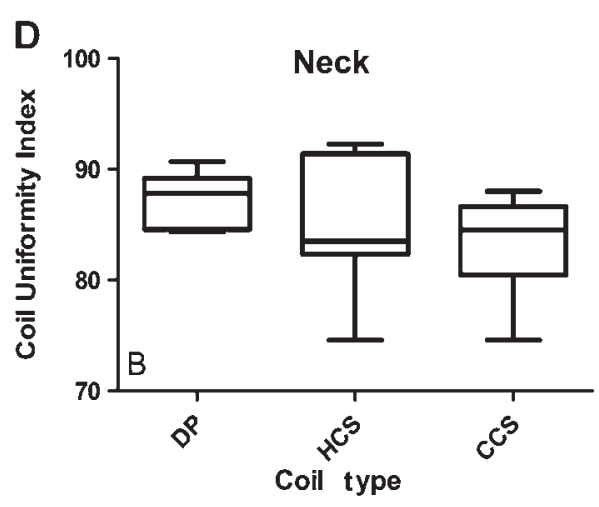


is a measure of the ability of the fluid to penetrate a given porous material, a phenomenon thought to be responsible for coil compaction. Permeability in the setting of coil embolization of brain aneurysms is a function of both the porosity (defined as the ratio of the void volume to total volume, an inverse measure of packing density) and coil uniformity. Compartmentalization of the coil mass with lack of uniform coil distribution is an important component of this phenomenon since it can produce regions permeable to blood flow.

The hypothesis of our research was that the design of the coil system could influence the coil distribution in an aneurysm model. The past decade of advancements in the detachable coil design has focused on the secondary coil shape. The often held claim that a particular coil type 'finds voids' has never been systemically quantified in preclinical models. To test this hypothesis, we employed systems of different secondary shapes, helical and complex coil systems, as well as the new Deltapaq system. The Deltapaq microcoil system is fundamentally unique in its design that the primary coil wind is altered from the traditional helix. This launches a new paradigm in coil technology that offers infinite combinations of primary coil wind and secondary shaping designs. Our results indicate that the Deltapaq achieves a tighter packing and a more homogenous distribution of the coil mass across the aneurysm dome in comparison with the traditional helical and complex coil systems, even though their surface area fractions are comparable. The Deltapaq also demonstrated the highest rate of angiographic occlusion based on the Raymond score. The Raymond score is a qualitative measure of permeability. Future studies may elucidate the specific relationship between coil uniformity, packing density and a quantitative measure of permeability. Our results taken in combination with the literature imply that achieving a higher uniformity of coil distribution along with a higher packing density as requisite parameters for coil selection may enhance the durability of endovascular treatment of cerebral aneurysms.

The use of in vitro models permits systematic comparisons between coil embolization using various microcoil systems by standardizing the variables experienced in in vivo studies. However, using simplified silicone side wall aneurysm models, deionized water as the circulating fluid and comparison of immediate post-embolization coil configuration are significant limitations of this study as they do not account for the anatomic variations of the cerebral vasculature, intra-aneurysmal thrombosis and the biological and hemodynamic response to the coil mass. ${ }^{14}$ The translation of uniform coil distribution in enhancing treatment durability remains a hypothesis and, at present, can only be indirectly inferred from long term treatment outcomes. Although we have tested three different coil types from the same vendor, the focus of our study was to assess how the intrinsically unique design properties of the three coils influences these parameters. To ensure the integrity and scientific merit of the study, maintain equipoise and demonstrate proof of concept we thought it prudent to select three different generations of coils from the same vendor. We realize the implications of this work in driving innovation in the biomedical device industry would be well served by testing these parameters in coils from other vendors in a publically funded trial.

\section{CONCLUSION}

Advances in biomedical engineering have made available newer coil designs which enable a more robust embolization of intracranial aneurysms. Introduction of coil uniformity index may replace the sole reliance on packing density as the surrogate

\section{Key messages}

- Coil compaction remains an impetus for technology development to improve the durability of aneurysm embolization.

- Both angiographic occlusion and packing density as surrogate parameters for treatment durability overlook regions of low coil density which can provide a nidus for the coils to compact.

- Previous work on modeling the interaction between the coils within an aneurysm with the hemodynamic stress suggests that the lower permeability of a coil mass at a given packing density may accelerate intra-aneurysmal thrombosis due to the reduction in intra-aneurysmal velocities and increased residence times.

- This implies that a homogenous distribution of the coil mass, a function of permeability, even at the same packing density, may enhance its performance and decrease aneurysm recanalization due to compaction.

- We have devised a repeatable in vitro assessment tool to objectively measure the coil uniformity index which systemically quantifies the ability of the different coil designs to 'find voids' in addition to the standard benchmarks of angiographic occlusion and packing density.

parameter in predicting treatment stability. We have devised a repeatable in vitro assessment tool to objectively compare coils for the standard benchmarks of angiographic occlusion and packing density, in addition to coil uniformity index. Application of this experimental model to evaluate other coil types in measuring their packing density and homogeneity can provide valuable information regarding their behavior within an aneurysm.

Funding This work was supported by a research grant from Micrus Endovascular (to $B R B$ and $M J G)$.

Competing interests MJG has been a consultant per hour for Micrus Endovascular Codman Neurovascular and Soteira Inc; receives research support from the National Institutes of Health, Guerbet, Micrus Endovascular Inc, Codman Neurovascular, Boston Scientific, eV3, Philips Healthcare, NeuroVasx Inc and Concentric Medical. FEL was formerly an employee of Micrus Endovascular. EIL receives research grant support, other research support (devices) and honoraria from Boston Scientific and research support from Micrus Endovascular; has an ownership interest in Intratech Medical Ltd and Mynx/Access Closure; serves as a consultant on the board of scientific advisors to Codman Neurovascular Cordis Corporation; serves as a consultant per project and/or per hour for Micrus Endovascular, ev3 and TheraSyn Sensors, Inc; and receives fees for carotid stent training from Abbott Vascular and ev3. BRB receives research support from Microvention, Brain Aneurysm Foundation and the Erika Keeney Foundation.

Provenance and peer review Not commissioned; externally peer reviewed.

\section{REFERENCES}

1. Molyneux A, Kerr R, Stratton I, et al. International Subarachnoid Aneurysm Trial (ISAT) of neurosurgical clipping versus endovascular coiling in 2143 patients with ruptured intracranial aneurysms: a randomised trial. Lancet 2002;360:1267-74.

2. Molyneux AJ, Kerr RS, Yu LM, et al. International subarachnoid aneurysm trial (ISAT) of neurosurgical clipping versus endovascular coiling in 2143 patients with ruptured intracranial aneurysms: a randomised comparison of effects on survival, dependency, seizures, rebleeding, subgroups, and aneurysm occlusion. Lancet 2005;366:809-17.

3. Raymond J, Guilbert F, Weill A, et al. Long-term angiographic recurrences after selective endovascular treatment of aneurysms with detachable coils. Stroke 2003; 34:1398-403.

4. Molyneux AJ, Kerr RS, Birks J, et al. Risk of recurrent subarachnoid haemorrhage, death, or dependence and standardised mortality ratios after clipping or coiling of an intracranial aneurysm in the International Subarachnoid Aneurysm Trial (ISAT): long-term follow-up. Lancet Neurol 2009;8:427-33. 
5. Raymond J, Darsaut T, Salazkin I, et al. Mechanisms of occlusion and recanalization in canine carotid bifurcation aneurysms embolized with platinum coils: an alternative concept. AJNR Am J Neuroradiol 2008;29:745-52.

6. Murayama Y, Nien YL, Duckwiler G, et al. Guglielmi detachable coil embolization of cerebral aneurysms: 11 years' experience. J Neurosurg 2003;98:959-66.

7. Sluzewski M, van Rooij WJ, Slob MJ, et al. Relation between aneurysm volume packing, and compaction in 145 cerebral aneurysms treated with coils. Radiology 2004;231:653-8.

8. Kole MK, Pelz DM, Kalapos $\mathrm{P}$, et al. Endovascular coil embolization of intracranial aneurysms: important factors related to rates and outcomes of incomplete occlusion. $J$ Neurosurg 2005;102:607-15.

9. Cha KS, Balaras E, Lieber BB, et al. Modeling the interaction of coils with the local blood flow after coil embolization of intracranial aneurysms. J Biomech Eng 2007:129:873-9.
10. Bendok BR, Parkinson RJ, Hage ZA, et al. The effect of vascular reconstruction device-assisted coiling on packing density, effective neck coverage, and angiographic outcome: an in vitro study. Neurosurgery 2007;61:835-40.

11. Chueh JY, Wakhloo AK, Gounis MJ. Neurovascular modeling: small-batch manufacturing of silicone vascular replicas. AJNR Am J Neuroradiol 2009:30:1159-64.

12. Roy D, Milot G, Raymond J. Endovascular treatment of unruptured aneurysms. Stroke 2001;32:1998-2004.

13. Piotin $\mathbf{M}$, lijima $\mathrm{A}$, Wada $\mathrm{H}$, et al. Increasing the packing of small aneurysms with complex-shaped coils: an in vitro study. AJNR Am J Neuroradiol 2003;24:1446-8.

14. Piotin M, Mandai S, Sugiu K, et al. Endovascular treatment of cerebral aneurysms: an in vitro study with detachable platinum coils and tricellulose acetate polymer. AJR Am J Roentgenol 2001;176:235-9.

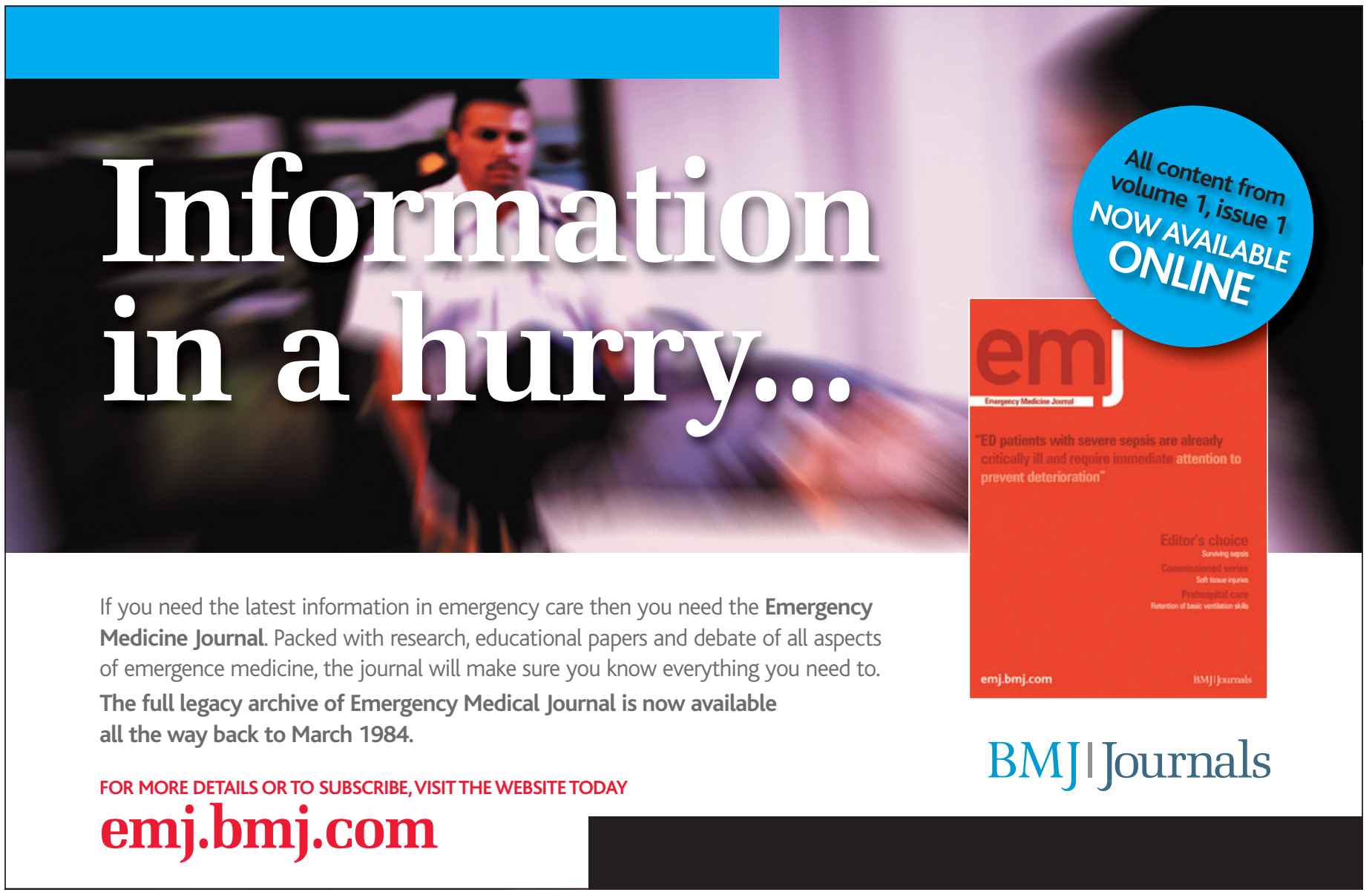

